\title{
Auditory sensory memory and working memory skills : Association between frontal MMN and performance scores
}

\section{Bonetti, L.}

2018-12-01

Bonetti , L , Haumann , N T, Brattico , E , Kliuchko , M , Vuust , P , Särkämö , T \& Näätänen , R 2018 , ' Auditory sensory memory and working memory skills : Association between frontal MMN and performance scores ' , Brain Research , vol. 1700 , pp. 86-98 . https://doi.org/10.1016/j.brainres.20

http://hdl.handle.net/10138/311679

https://doi.org/10.1016/j.brainres.2018.06.034

cc_by_nc_nd

acceptedVersion

Downloaded from Helda, University of Helsinki institutional repository.

This is an electronic reprint of the original article.

This reprint may differ from the original in pagination and typographic detail.

Please cite the original version. 


\title{
Auditory sensory memory and working memory skills: Association between frontal MMN and performance scores
}

Bonetti, L.*, Haumann, N. T.*, Brattico, E.*, Kliuchko, M.* , Vuust, P.*, Särkämö, T.^, Näätänen, R. ${ }^{\circ}$

*Center for Music in the Brain, Department of Clinical Medicine, Aarhus University, \& The Royal Academy of Music Aarhus/Aalborg, Denmark

${ }^{\wedge}$ Cognitive Brain Research Unit, Department of Psychology and Logopedics, Faculty of Medicine, University of Helsinki, Finland

1 BioMag Laboratory, HUS Medical Imaging Center, University of Helsinki and Helsinki University Hospital, Finland Institute of Psychology, University of Tartu, Tartu, Estonia

Contact information for the corresponding author: Leonardo Bonetti, PhD fellow at Center for Music in the Brain (MIB), Department of Clinical Medicine, Aarhus University, \& The Royal Academy of Music Aarhus/Aalborg, Denmark, Nørrebrogade 44, Aarhus. Email: leonardo.bonetti@clin.au.dk. Telephone: 00393346150761

\begin{abstract}
:
Objective: Memory is the faculty responsible for encoding, storing and retrieving information, comprising several subsystems such as sensory memory (SM) and working memory (WM). Some previous studies exclusively using clinical population revealed associations between these two memory systems. Here we aimed at investigating the relation between modality-general WM performance and auditory SM formation indexed by magnetic mismatch negativity (MMN) responses in a healthy population of young adults.
\end{abstract}

Methods: Using magnetoencephalography (MEG), we recorded MMN amplitudes to changes related to six acoustic features (pitch, timbre, location, intensity, slide, and rhythm) inserted in a 4-tone sequence in 86 adult participants who were watching a silent movie. After the MEG recordings, participants were administered the WM primary subtests (Spatial Span and Letter Number Sequencing) of Wechsler Memory Scale (WMS).

Results: We found significant correlations between frontal MMN amplitudes to intensity and slide deviants and WM performance. In case of intensity, the relation was revealed in all participants, while for slide only in individuals with a musical background.

Conclusions: Automatic neural responses to auditory feature changes are increased in individuals with higher visual WM performance.

Significance: Conscious WM abilities might be linked to pre-attentive sensory-specific neural skills of prediction and short-term storage of environmental regularities.

Keywords: Working memory (WM); Sensory memory (SM); Mismatch negativity (MMN); Magnetoencephalography (MEG); Wechsler Memory Scale (WMS). 


\section{Introduction}

Memory is the faculty responsible for encoding, storing and retrieving information (Förstl et al., 2006) and represents one of the most crucial abilities for surviving and acting within the environment (Kumar et al., 2015). It comprises several sub-systems, such as sensory memory (SM) and working memory (WM), that together concur in processing the information. SM is the memory system that allows individuals to retain traces of sensory information after that the original stimulus has terminated (Cowan, 2010). It is strongly involved in the processing of sensory stimuli and orienting within the environment, and it is active in every moment of an organism's life. Taken in by sensory receptors and processed by the nervous system, sensory information is stored in SM just long enough to be transferred to short-term memory and used in WM (Cowan, 2010; Sligte et al., 2010).

WM is a conscious cognitive system with limited capacity and responsible for the transient holding, processing, and manipulation of information. It plays a fundamental role in cognitive processes, such as reasoning and decision making (Baddeley, 1992; Förstl et al., 2006). In the past decades, several theories have been formulated to describe and explain its function. One of the most important theories was conceived by Baddeley and Hitch (1974) and further developed by Baddeley (2000). They proposed a multi-component model of WM, comprising four constituents that process and integrate auditory and visual information: the central executive, the phonological loop, the visual-spatial sketchpad and the episodic buffer.

Another theoretical approach was introduced by Cowan (1999) who highlighted a connection between WM and attention, conjointly controlled by voluntary and involuntary processes. This perspective considered WM to be organized into embedded levels comprising long-term memory and a subset of activated memory traces that are in the focus of attention and awareness (Cowan, 1999). In this framework, attention plays a fundamental role in selecting and retaining information for cognitive purposes. In addition, as reported by other literature, the amount of attention that individuals invest in WM tasks has been associated with the cognitive performance, meaning that a higher attention level corresponds to an improved performance in cognitive abilities (Awh et al., 2006). This finding has been corroborated by a large cohort of behavioural and neuroscientific studies that described the crucial role of attention for WM demands, specifically in the encoding and maintenance of information (Chun, 2011; Duarte et al., 2013; Fougnie, 2008; Oberauer \& Hein, 2012). Even if it appears less clear and still debated, attention seems to be connected also to SM. Previous studies, for instance, reported evidence of positive correlations between attention and early neurophysiological responses usually associated with SM, such as P50 (Cowan, 2008; Lijffijt et al., 2009).

A relevant contribution focusing on the relationship between WM and SM has been offered by Pasternak and Greenlee (2005). In their review, the authors defined the sensory WM as a short-term storage of sensory stimuli adapted to guide behaviour, asserting the importance of this system for a precise sensory encoding and for the formation of a short-term information storage. Specifically, they stated that elemental sensory objects, such as basic visual or auditory stimuli, are stored by segregated feature-selective systems. Those systems do not comprise only the prefrontal and parietal cortex but also regions of sensory cortex that are involved in early stages of brain processing of stimuli, thus supporting two functions: precise sensory encoding and short-term storage of the information. Therefore, in their work, Pasternak and Greenlee (2005) suggest the existence of common functional neural basis for WM and SM. 
Other studies proposed not only WM, but also general intelligence as able to optimize and influence the detection of sensorial stimuli. Deary et al. (2004) showed a connection between the level of intelligence and the accuracy of sensory discrimination, while Acton and Schroeder (2001) reported a weak but significant connection between general intelligence and sensory discrimination of pitch and colour.

In our study, to better explore the mechanisms underlying detection, retention and manipulation of information, we focused on the relationship between the neurophysiological evidence of SM formation and the individual WM abilities. A reliable neurophysiological index of SM formation is the event-related potential (ERP) component known as mismatch negativity (MMN) (Atienza et al., 2002; Cheour et al., 2000; Ruzzoli et al., 2012; Winkler \& Czigler, 2012), which forms predictions of the incoming sounds and detects deviations from them (Näätänen et al., 1978). The $M M N$ is elicited by a deviant stimulus inserted in a sequence of repetitive coherent stimuli with respect to one or multiple sound features, such as pitch, location, rhythm, intensity, timbre, or even abstract simple auditory rules (Näätänen, 1992; Näätänen et al., 2011). Several studies showed a higher sensitivity to changes along different acoustic important dimensions indexed by MMN in individuals with superior musical skills, typically due to formal training (Bonetti et al., 2017; Brattico et al., 2009; Brattico et al., 2001; Garza Villarreal et al., 2011; Vuust et al., 2012; Vuust et al., 2016). These findings suggest that MMN provides a tool for the assessment of auditory SM formation as well as an index of its improvement with training or exposure to certain stimulation (Näätänen et al., 2014).

Auditory MMN originates from generators located within the temporal and frontal cortex. Specifically, temporal generators have been shown to be involved in the detection of sound change, while the frontal ones have been associated with the involuntary switching of attention due to the sound deviation (Deouell, 2007; Rinne et al., 2000; Tse \& Penney, 2008). Other evidence suggested a relevant connection between attention and frontal MMN generators. Escera et al. (1998), for instance, associated frontal MMN with the automatic orienting response toward an acoustic change, while Opitz et al., (2002) described the frontal MMN generators as responsible for an involuntary amplification mechanism able to tune the auditory change detection system. In another study, Restuccia et al. (2005) recorded auditory $\mathrm{MMN}$ while participants were required to perform an attentional visual task characterized by different levels of attentional demand. They discovered that, while the temporal generators were not influenced by the visual task, the right frontal generators were active only for the high-load attentional condition of the visual task. Furthermore, some studies pointed out that attention differentially modulates the MMN depending on the deviating feature. The MMN response to intensity, for instance, decreases in the absence of attention, whereas the MMN for pitch and timbre are independent of attentional load, as shown by Näätänen et al., (1993) and Takegata (2005), respectively.

MMN and memory systems have also been studied in cases of memory function impairments, or taking into account individual differences in auditory skills. Javitt et al. (1995), for instance, reported impaired MMN in patients with schizophrenia, while Hahn et al. (2012) Park \& McTigue (1997) Potkin et al. (2009) described lower WM performance in schizophrenic patients compared to healthy controls. This evidence highlights the broad memory dysfunction in patients with schizophrenia, suggesting that the impairment of memory and executive processes in this disorder can be observed at both low and high levels, giving strength to the idea that memory is a continuous system characterized by a close interaction between its subsystems such as SM and WM. 
In another study, Ahveninen et al. (1999) reported a WM impairment in alcoholics as indexed by MMN, suggesting that the vulnerability to memory trace disruption by shortly following sounds (according to the backward masking paradigm) may be one of the factors contributing to behavioural memory dysfunction in alcoholics. Other studies underlined the key role of the MMN in indexing auditory SM. Recently, Näätänen et al., (2014) proposed MMN as a possible tool to study a large variety of disorders related to cognitive abilities involving the auditory domain, underlying the potential role of MMN in indexing cognitive deficits related to hearing. Several other studies showed that the individual behavioural auditory discrimination accuracy can be predicted by the sensitivity of the MMN response to small stimulus changes, both for linguistic (Cheour et al., 2002; Kraus et al., 1995; Tremblay et al., 1997; Winkler et al., 1999) and non-linguistic stimuli (Näätänen et al., 1993; Tervaniemi, 2001).

Previous literature described the MMN as a reliable index for detecting SM formation, defining the differential roles of its frontal and temporal generators in involuntary switching of attention and change detection, respectively. MMN has been shown able to identify differences in the stimulus characteristics after their presentation, becoming an index of the automatic change detection system in the brain and working closely with the auditory sensory memory (ASM) (Näätänen, 1990). This evidence suggests a close relationship between SM and MMN, giving strength to the idea that memory is constituted by different sub-systems that cooperate together. Furthermore, previous studies described general associations between impaired cognitive abilities and reduced MMN responses in clinical populations. Based on this prior evidence, in this paper we aimed at exploring the relationship between auditory SM formation indexed by MMN and overall WM capacity in a healthy population. Thus, we analysed WM performance as a predictor of MMN, hypothesizing larger frontal MMN responses to sound deviants in participants who demonstrate better performance in WM tasks. We focused our analysis mainly on the frontal and temporal regions of the brain because they are strongly involved in the WM and attentional tasks (Courtney et al., 1998; McNab \& Klingberg, 2008). Particularly, frontal MMN generators have previously been shown to be related to involuntary attention switch to novel, unexpected sound events (Deouell, 2007; Rinne et al., 2000; Tse \& Penney, 2008). Therefore, in this study we aimed to suggest a relationship between the level of conscious cognitive capacities, such as WM, and the automatic neural discrimination of sound changes.

Moreover, considering that WM is a cognitive system responsible for manipulation of information coming from different sensory modalities (Baddeley, 1992; Suchan et al., 2006), in our study we opted to use auditory stimuli to record $\mathrm{MMN}$ and neuropsychological tests using both visually and verbally presented items to assess conscious use of WM. This procedure allowed us to explore the relationship between SM and WM involved in the processing of information coming from different sensory modalities. Finally, we performed additional stratified analysis according to the musicianship of the participants. Considering that previous literature showed the relevance of musical background on auditory discrimination and music processing (Brattico et al., 2009; Vuust et al., 2011; Vuust et al., 2012) as well as higher WM performance in musicians compared to non-musicians (Hansen, Wallentin, \& Vuust, 2013; Talamini, Carretti, \& Grassi, 2016), we hypothesized to reveal stronger relationships between MMN and WM in musicians than in nonmusicians.

\section{Results}




\subsection{MMN and standard responses}

Independent t-tests have been performed comparing the event-related fields (ERFs) in response to deviants with those in response to standards. Specifically, we have employed one t-test per deviant type, comparing those deviants with all of the standards comprised in the musical multi-feature paradigm (mumufe) (Table 1). Every t-test was significant. Waveform figures are depicted in Figure 1.

\section{$2.2 \quad M M N$ and working memory}

The multivariate test of the MANCOVA involving frontal sensors was significant for the Working Memory Index: $F(1,85)=3.00, p=.01, \eta^{2} p=.18$, but not for the musical membership: $F(1,85)=1.42, p=.19, \eta 2 p=.10$. The effect of age as covariate was also significant: $F(1,85)=4.59, p<.001, \eta 2 p=.26$. The MANCOVA for the temporal ROI showed a nonsignificant effect for Working Memory Index: $F(1,85)=.93, p=.48, \eta 2 p=.07$ and for the musical membership: $F(1,85)=$ 2.06, $p=.07, \eta^{2} p=.14$.

Specifically, the first MANCOVA highlighted the significant relationship between WM and MMN to two deviants, intensity and slide, as reported in Table 2. Waveforms and Pearson's correlation plots for intensity and slide are depicted in Figure 2 and Figure 3, respectively.

\subsection{Intensity and slide deviants}

Regarding intensity deviant, the two regressions calculated for both musicians and non-musicians showed a similar effect (Table 3, Figure 4).

For slide deviant in contrast, the regression was significant only for musicians and not for non-musicians (Table 4, Figure 5).

\subsection{Spatial Span and Letter Number Sequencing in relation to MMN}

We found a significant main effect for the Spatial Span subscale on frontal MMN amplitude (Multivariate test: $\left.F(1,85)=2.23, p<.05, \eta_{p}^{2}=.14\right)$, while the musical membership did not have a significant effect: $F(1,85)=1.44, p=.21$, $\eta_{p}^{2}=.10$. As observed for the general Working Memory Index, both intensity and slide were significant (Table 5). On the contrary, this analysis showed a non-significant effect for every deviant for Letter Number Sequencing (Multivariate test: $F(1,85)=.22, \mathrm{p}=.97, \eta 2 \mathrm{p}=.02)$. The effect of age as covariate was also significant: $F(1,85)=4.51, \mathrm{p}=.001, \eta 2 \mathrm{p}=$ .26 . 
This study reports enhanced MMN responses to auditory feature deviants in individuals with higher performance in WM, suggesting a link between the accuracy of auditory SM trace formation and overall WM capacity. We found higher amplitude of the frontally located MMN to intensity and slide deviants in participants with higher WM abilities. Furthermore, the same analysis performed on the MEG sensors located above temporal brain sites did not yield any significant relationship with the overall WM scores. Considering each WM subscale score separately, we found a significant correlation with MMN for Spatial Span but not for Letter Number Sequencing. Furthermore, separate analyses looking at musical expertise of the participants showed different results for the slide deviant, but not for the intensity one. Specifically, the relationship between MMN amplitudes to slide and Working Memory Index emerged only in the sample of musicians, indicating that musicians who scored better in WM tasks exhibited higher MMN to slide.

The principal aim of our study was to understand the relationship between the formation of auditory SM traces and WM abilities, hypothesizing a link between the automatic, pre-attentive adaptation of the auditory cortex and the conscious WM performance. As a development of the previous theories (see Baddeley, 1992; Cowan, 1999), Pasternak and Greenlee (2005) extended the nature of WM to the sensory domain, asserting its importance for a precise sensory encoding and for the formation of a short-term information storage, suggesting the existence of common functional neural basis for WM and SM. In accord with their proposal, other studies provided evidence that suggested a link between sensory and working memory in showing impaired auditory MMN in clinical populations who reported also WM deficits (Ahveninen et al., 1999; Javitt et al., 1995). In a review, Näätänen et al. (2010) proposed that automatic sensory-cognitive processes might represent a basis for higher-order cognitive processes, suggesting MMN as a neural index of automatic sensory predictions in the short-term span. They reported that, surprisingly, complex processes in the auditory domain, such as stimulus anticipation and extrapolation, sequential stimulus-rule extraction, pattern and pitch-interval encoding, happen automatically and mainly in the sensory-specific cortical regions. In a recent MEG study, Cashdollar et al. (2016) demonstrated that when visual stimuli occurred in a regular time series, individuals who performed better in WM tasks displayed enhanced slow-wave neural oscillations in the theta band prior to the stimulus onset, influencing the sensory processing of the following visual information. Even if this research targeted aims that differed from ours, both Cashdollar et al. (2016) and our study described a link between WM performance and both pre-attentive sensory processing and change detection system. Moreover, consistently with our findings, other MMN studies showed relationships between memory, attention and MMN responses to sound and visual deviants, proposing MMN as an ERP component that indexes the rapid and stimulus-specific adaptation that underlies SM formation in the auditory cortex (Winkler \& Czigler, 2012, Haenschel et al., 2005).

As a priori hypothesized, in this study we revealed a significant relationship between MMN amplitude and WM performance exclusively for the frontal areas of the brain, reporting higher brain responses connected to the WM performance for intensity and slide deviants. Our hypothesis on the frontal MMN was driven by the large recruitment of frontal brain areas during WM tasks (Courtney, 1998; Courtney et al., 1998; McNab \& Klingberg, 2008; Onton et al., 2005) and by the role of attention that is relevant for both WM abilities and frontal MMN (Deouell, 2007; Escera et al., 1998; Opitz et al., 2002; Rinne et al., 2000; Tse \& Penney, 2008). Furthermore, the detection of the deviants in an ever- 
changing auditory sequence (as the one used in the musical multi-feature paradigm) involves a discrimination of the physical characteristics of the sounds and a memory process for detecting the mismatch between the standard and the deviant stimuli. The temporal areas of the brain, especially of the right hemisphere, are mainly involved in the discrimination of the physical sound features (Tervaniemi, 2006) and therefore they might be not related to WM processes underlying digit span tasks. The frontal MMN response in contrast, which appears to be related to the involuntary switching of attention toward the deviant (Deouell, 2007; Rinne et al., 2000; Tse \& Penney, 2008), might be remarkably connected to high-level memory processes, such as WM, that involve frontal regions of the brain (McNab \& Klingberg, 2008; Onton et al., 2005).

Interestingly, we revealed a significant relationship between WM and MMN to intensity change, a deviant that had previously been related to the attention paid by participants to the stimuli, which is an effect that was not found for the pitch deviant (Näätänen et al., 1993). The current relationship between frontal MMN and Spatial Span subscale score might be ascribed to the specific involvement of frontal areas of the brain during spatial WM tasks, as showed by Courtney et al., (1998), Curtis (2006) and Onton et al. (2005).

Our results showed a significant relationship between MMN and the Spatial Span subscale, while the Letter Number Sequencing score was not related to MMN responses. This discrepancy might be ascribed to the different nature of the two subscales. The Letter Number Sequencing task involves a high-level cognitive manipulation of the information but rather low-level auditory SM processes, while the Spatial Span subscale measures the individual's ability to hold a visual-spatial sequence of information, a task that is more related to the visual SM (Wechsler, 1997). This observation might explain the connection between the MMN responses to unexpected sensory changes in auditory stimulation and the visual Spatial Span subscale.

The Working Memory Index assessed in this study has been obtained by administering not only an auditory task but also a visual one, while the deviant stimuli eliciting the MMN responses were auditory. The choice of utilizing tasks involving two different sensory modalities was motivated by the hypothesized possible sensory-general nature of the WM. Previous literature showed that WM is designated to process and manipulate information deriving from different sensory modalities (Baddeley, 1992; Baddeley, 2012; Suchan et al., 2006) and that auditory perception and memory are connected to other sensory domains (Arnell, 2006; Bonetti \& Costa, 2017b; McKone \& Dennis, 2000). Furthermore, Allen et al. (2009), studying WM and attention during cross-modal tasks, found no impairment in the case of information coming from different sensory modalities. Those results suggested that WM might be a system which is able to integrate cross-modal information without necessarily causing a decrement of the performance. In a recent study, Constantinidis (2016) reported that neurons in the pre-supplementary motor area encoded both tactile and auditory information involved in WM tasks, using a shared representation for both sensory modalities. This piece of evidence adds a further contribution to support the hypothesis that neural substrates of WM might have a sensorygeneral nature and integrate information coming from different sensory modalities.

Another relevant finding here lies in the mediation of the MMN and WM relationship operated by musical expertise. Performing separate analyses for musicians and non-musicians, we noticed that the relationship between MMN to slide and WM was significant only for musicians, while we reported no differences according to the musical expertise in the case of intensity deviant. This finding is consistent with a number of previous studies describing 
differences in auditory MMN responses between musicians and non-musicians (Brattico et al., 2009, 2001; Brattico et al., , 2006; Garza Villarreal et al., 2011). The revealed difference found for slide but not for intensity might be ascribed to the nature of the two deviants. Intensity is a sound feature related not only to music but present in all sounds and noises occurring in everyday life (Fahy, 1997; Gifford et al., 2011). Furthermore, Western musical training does not focus on intensity as strongly as on pitch, intonation and slide from one note to another. Particularly, slide introduces notes that are not in tune with the well-tempered scale. For this reason, musicians, who often spend time learning how to be perfectly in tune, might be more sensitive to this kind of deviation, which is, in contrast, not salient in language. This notion is consistent with Koelsch et al., (1999) and Tervaniemi et al., (2005) who found that musicians, violinists in particular, exhibited higher MMN to deviants related to slide and intonation. In another study, Moreno et al. (2009) showed the beneficial effect of musical training on the discrimination of pitch in both music and speech prosody in 8year-old children. This consideration might explain why in the present study, the relationship between MMN and WM performance was significant only for musicians in case of slide, while no differences due to musicianship emerged for the intensity deviant. In consideration of this dissociation and noticing relevant connections between cognitive abilities and music perception (Bonetti \& Costa, 2016, 2017a), future studies could explore possible links between music perception and WM performance, also taking into account the musicianship of the participants.

Our study highlighted a connection between auditory sensory memory traces indexed by MMN to auditory feature changes and WM abilities, implying a number of future perspectives. For instance, on the basis of these results it could be argued that a first evidence of intelligence and conscious cognitive abilities might come even from a preattentive sensorial level. This hypothesis would be consistent with Deary et al. (2004) who showed a connection between the level of intelligence and the accuracy of sensory discrimination and with Lindenberger and Baltes (1994) who reported the same relationship, found in a particular sample composed by people older than 80 . The higher sensitivity of the brain to fast sound-related changes could be seen as a primary skill that enables humans to predict future events and detect errors, abilities clearly connected to high-order cognitive abilities (Deary, 2012). Furthermore, the adaptive formation of SM traces for repetitive aspects of the auditory environment, in this study indexed by MMN, might enable us to keep information in a short-term storage, allowing a successive further elaboration of the WM. Moreover, to deeply explore the nature of WM in terms of contribution from different sensory modalities on the overall WM capacity, follow-up research based on our study might assess the relationship between WM and MMN responses to other modality deviants, such as visual or tactile.

\section{$4 \quad$ Methods and materials}

\subsection{Participants and Working Memory assessment}

We recruited 86 adult well-educated Finnish participants, 36 males (41.9\%) and 50 females (58.1\%). Their mean age was $28.4 \pm 8.6$, and they were 79 right-handed and 7 left-handed. Furthermore, the sample was composed of 44 musicians and 42 non-musicians. Musicians had several years of professional and amateur musical training in addition to the general musical education received at school $(9.2 \pm 8.1)$, while non-musicians had not received more than 3 years 
of musical training outside the school curriculum (Table 6). The participants were compensated with vouchers for cultural and sport purposes (e.g. concerts, museums or swimming pools). All participants were healthy and not under medication. They declared to have normal hearing and did not report any neurological or psychiatric problems occurred in their past. All participants signed an informed consent upon arrival to the laboratory, and a researcher was present and available for assistance. All experimental procedures for this study, included in the larger research protocol called "Tunteet", were approved by the Coordinating Ethics Committee of the Hospital District of Helsinki and Uusimaa (approval number: 315/13/03/00/11, obtained on March the 11th, 2012). Furthermore, all procedures were conducted in agreement with the ethical principles of the Declaration of Helsinki. Part of the dataset was analysed (using different analysis software, BESA and FieldTrip, and therefore following slightly different pipelines) for studying individual differences in noise sensitivity and in depression trait and reported in two previous papers (Kliuchko et al., 2016; Bonetti et al., 2017). The datasets are not fully overlapping since not all participants could take part in the different experimental sessions. A third paper on a small part of this dataset (Haumann et al., 2016) reported methodological improvements in the analysis pipeline that led up to the renewed version adopted by Bonetti et al., 2017 and employed in this current paper.

The Wechsler Memory Scale-III (WMS-III) is one of the most used scales for evaluating memory (Hoelzle et al., 2011) and comprises three primary indexes, known as Immediate Memory, General Memory, and Working Memory (Wechsler, 1997). In this study, to assess the working memory (WM) level of each participant, we administered the two primary subtests of Working Memory Index, namely Spatial Span and Letter Number Sequencing. These WM tasks involve concentration, attention, sequential processing, mental manipulation, memory span, and short-term memory. Furthermore, it has been shown that attention is a strong predictor of WM performance and high-level cognition, and that the resolution of WM tasks implies a sustained use of executive attention (Engle, 2002; Engle \& Kane, 2004; Mccabe et al., 2010). WM tasks are also related to cognitive flexibility, information processing, and fluid intelligence (Crowe, 2000; Kaufman \& Lichtenberger, 2006; Kaufman et al., 2008). According to Wechsler (1997), the Spatial Span loads on the Working Memory Index as well as the Letter Number Sequencing subscale, even if they are not related to each other (Wilde \& Strauss, 2002).

The Spatial Span subtest is a visual task composed by two parts: Spatial Span Forward and Spatial Span Backward. For each task, the examiner taps a series of cubes, touching approximately one cube per second. After the presentation, the examinee is requested to tap the cubes in the same order observed by the examiner for the Spatial Span Forward task, while in the reverse order in the Spatial Span Backward. For both tasks, the test begins with a series of two cubes and proceeds to a maximum of eight cubes. The participant is given two trials at each series length, and the test continues until both trials are failed. Otherwise, in case of at least one correct response per series the test proceeds. The participant is awarded one point for each correct trial. The maximum possible score for the Spatial Span subtest is 32 (16 points each for Spatial Span Forward and Backward).

In the Letter Number Sequencing subscale, the experimenter says a list of alternating numbers and letters at the rate of about one per second (e.g., L, 7, 3, A). After the presentation, the participant is requested to report first the numbers in ascending numerical order, and then to enunciate the letters in alphabetical order (e. g., 3, 7, A, L). Therefore, the task involves auditory information that participants are requested to memorize. The test begins with series of two items (one number and one letter) and continues progressively increasing the number of items, up to a maximum of eight items (four numbers and four letters). The participant is given three trials for each series length, and 
the examination proceeds until all three trials of a given length are failed. The maximum possible score is 21 . Finally, the global Working Memory Index is calculated combining the two subscales scores. The raw scores are then converted into age-adjusted standard scores following the tables of conversion reported in the manual (Wechsler, 1997). Values obtained from the Working Memory Index, and for the other WMS-III primary indexes, are standard scores with mean $=100$ and $S D=15$ (while subscales yield scaled scores with mean = 10 and SD = 3) (Lichtenberger \& Smith, 2005). Standardized scores for Spatial Span, Letter Number Sequencing, and Working Memory Index are reported in Table 7. A univariate ANOVA showed no significant difference between the scores reported by musicians and non-musicians (Spatial Span: $p=.14$; Letter Number Sequencing: $p=.50$; Working Memory Index: $p=.16$ ).

\subsection{Procedure and stimuli}

The subjects' background questionnaires were administrated before the preparation for the MEG/EEG recordings, while the WM subscales of WMS-III were performed on a separate day before or after the MEG/EEG measurement. In the MEG/EEG recordings, the participants were instructed to passively listen to auditory sequences through headphones (Sennheiser HD 210; Sennheiser electronic GmbH \& Co. KG, Wedemark-Wennebostel, Germany). The sound volume was adjusted prior to the recording to a level that was comfortable for the participant. We requested the participants to concentrate on a silent movie while sitting comfortably in a shielded chamber. Head position indicator coils (HPI) were used, measuring the head position of the participant before each recording.

We used the musical multi-feature MMN paradigm (Vuust et al., 2011) as stimuli (Figure 6). This paradigm provides the listener with different sound-feature changes embedded in a musical sounding context and is known to elicit reliable MMN (Vuust et al., 2011; 2012; 2016). The paradigm is created by inserting different feature changes in a four-tone pattern called the Alberti Bass: an accompaniment commonly used in the Western music. In this study, we used a modified version of the musical multi-feature MMN paradigm with six types of deviations, one presented in every pattern, as opposed to a presentation of one in every second pattern in the original version, reducing the duration of the experiment to only 12 minutes. Stimuli were piano tones from the Wizoo Acoustic Piano sample sounds from the software sampler Halion in Cubase (Steinberg Media Technologies GmbH, Hamburg, Germany) and peak amplitude was normalized using Audition, Adobe Systems Incorporated (C). We used peak amplitude normalization, as it allows for balancing sounds based on their most salient portion, namely the sharp attack. Each piano tone was 200 ms in duration with $5 \mathrm{~ms}$ of raise and fall time with an interval of $5 \mathrm{~ms}$ between the stimuli. The musical key of the presentation changed after every six patterns in pseudorandom order. The keys were kept in the middle register. 24 keys were used and varied according to musical mode (12 major and 12 minor). In each pattern, the third tone was replaced with one of the six deviants: pitch, timbre, location, intensity, slide and rhythm. The deviant sounds were created by modifying one sound feature in Adobe Audition. The pitch deviant was obtained mistuning the third tone by 24 cents, tuned downwards in the major mode, and upwards in the minor mode. We used the "old-time radio" effect in Adobe Audition to create timbre deviant. The location deviant was created by decreasing the intensity in one of the audio channels that resulted in perceptual shift of the sound source location from the center to a side. The intensity deviant was a reduction of a sound intensity by $6 \mathrm{~dB}$. The slide deviant was obtained by a gradual change of pitch from one note below up to the standard tone over the duration of the sound. The rhythm deviant was made by shortening a tone by 60 ms but keeping 
the ISI of 5ms, resulting in the consequent tone arriving earlier than expected. Each deviant was presented 144 times, half of which (72) played in a major mode and the other half in a minor mode. Randomization was performed in Matlab (MathWorks, Natick, Massachusetts, United States of America), and the stimuli were presented using Presentation software (Neurobehavioural Systems, Berkeley, CA). Data acquisition

Simultaneous MEG and EEG data were collected at the Biomag Laboratory of the Helsinki University Central Hospital. The measurements were performed in an electrically and magnetically shielded room (ETS-Lindgren Euroshield, Eura, Finland) with Vectorview ${ }^{\mathrm{TM}}$ 306-channel MEG scanner (Elekta Neuromag ${ }^{\circledR}$, Elekta Oy, Helsinki, Finland) equipped with a compatible EEG system (EASYCAP GmbH, Herrsching, Germany). The MEG scanner had 102 sensor elements comprised of 102 orthogonal pairs of two planar gradiometer SQUID sensors and 102 axial magnetometer SQUID sensors. The EEG cap comprised 64 cap-mounted electrodes arranged according to the international 10-20 system. The reference electrode was placed on the nose tip and the ground electrode positioned on the right cheek. Blinks and vertical and horizontal eye movements were recorded with four electrodes attached above and below the left eye and close to both sides of the external eye corners. Four head position indicator coils were placed on top of the EEG cap. Their positions were located respectively to the nasion and the prearicular anatomical landmarks by Isotrack 3D digitizer (Polhemus, Colchester, VT, USA). MEG and EEG data were recorded with a sample rate of $600 \mathrm{~Hz}$. During the measurements, subjects watched a silent movie with subtitles. The stimuli were presented with Presentation software (Neurobehavioral Systems, Ltd.). Stimuli were delivered through a pair of pneumatic headphones.

\subsection{Data analysis}

\subsubsection{Data pre-processing}

We applied Elekta Neuromag ${ }^{\text {TM }}$ MaxFilter 2.2 (Elexta Oy, Helsinki, Finland) Temporal Signal Space Separation (tSSS) (Taulu \& Hari, 2009) with the default settings of inside expansion order of 8, outside expansion order of 3, automatic optimization of both inside and outside bases, subspace correlation limit of 0.980 , and raw data buffer length of 10 seconds, in order to minimize the influence of external and nearby noise sources and automatically detect and correct bad MEG channels. The subsequent data processing was performed with the open source toolbox for Matlab (Donders Institute for Brain, Cognition and Behaviour/Max Planck Institute, Nijmegen, the Netherlands) (Oostenveld et al., 2011) known as FieldTrip, version r9093, and Matlab R2013b (MathWorks, Natick, Massachusetts). On average one channel (range 0 to 10 channels) per subject was observed as bad and replaced by interpolations of the waveforms measured in the neighboring channels. The sampling rate was reduced from 600 to $300 \mathrm{~Hz}$, and high- and low-pass filters were applied with half cut-off frequencies at 1 and $25 \mathrm{~Hz}$, respectively, to analyze the MMN component of interest. The influence of artifacts, such as eye movements and cardiac activity, was reduced by applying Independent Component Analysis (ICA) with the logistic infomax algorithm implemented in the runica function for Matlab (Haumann et al.,, 2016; Makeig et al., 1996). The total number of removed artifactual ICA components per subject was on average 1.9 (range 0 to 3 components for the EEG, 2.6 (range 1 to 3 components) for the MEG magnetometers, and 2.6 (range 1 to 3 components) for the MEG gradiometers. Then, the two sensors within each couple of the MEG gradiometers were combined together using the Root-Mean-Square (RMS) algorithm. Successively, the data were epoched into responses 
to the six deviant types and standard trials, and a baseline from -100 to $0 \mathrm{~ms}$ pre-stimulus baseline was applied. According to the procedure followed in previous studies (Bonetti et al., 2017; Peltola et al., 2003) trials with artifacts with amplitudes exceeding $100 \mu \mathrm{V}, 2000 \mathrm{fT}$, or $400 \mathrm{fT} / \mathrm{cm}$ were rejected. Rejected trials were on average $3 \%$ from the EEG data, $0 \%$ from the MEG magnetometer data, and 2\% from the MEG gradiometer data, evenly distributed across deviant types and standard trials. The average standard response was then subtracted from the average deviant responses to isolate the MMN waveforms. The MMN peak latencies for each feature were estimated from the grand average of event-related field waveforms across all participants. The latencies corresponding to the peak MMN after the grand average across all participants were: 170 ms (intensity), 113 ms (location), 203 ms (pitch), 73 ms (rhythm), 180 ms (slide), $127 \mathrm{~ms}$ (timbre). The mean MMN amplitudes for each participant and channel were calculated by applying time windows of \pm 15 ms around the peak latencies for each feature.

\subsubsection{Statistical analysis}

We conducted statistical analyses exclusively for the MEG gradiometers because they have lower noise level than magnetometers, as documented by quantitative measures of signal-to-noise ratio for this dataset reported in Haumann et al. (2016).

In order to improve the reliability of our analysis, and following the guidelines provided by Gross et al. (2013), we decided to take into account an extensive number of frontal and temporal sensors, averaging the amplitudes that we recorded into two large regions of interest (ROIs) chosen around the peak amplitude channels (one frontal and one temporal, see Figure 7).

Firstly, to test the reliability of the musical multi-feature paradigm independent t-tests were performed comparing the event-related fields (ERFs) in response to deviants to those in response to standards. Specifically, we have employed one t-test per deviant type, comparing those deviants with all of the standards comprised in the paradigm. The analysis was performed for both frontal and temporal channels. After testing the reliability of the paradigm and MMN, in the following analysis we employed the amplitude of the MMN responses after subtracting the evoked response to the standards. Therefore, we performed two MANCOVAs, one for the temporal ROI and the other one for the frontal ROI. We inserted the Working Memory Index of the WMS-III as independent variable, the musicianship as grouping factor and the MMN amplitudes to each deviant as dependent variables. Moreover, we controlled for age, inserting it as covariate. Then we further explored the observed relationship by computing regressions involving only the significant deviants from the previous MANCOVAs. Since musicianship has been shown able to affect brain responses to auditory stimuli (Brattico et al., 2009; Vuust et al., 2011; Vuust et al., 2012), we performed separate regressions for the two musically training groups involved in this study, in order to evaluate whether musical expertise would affect the relationship between MMN and WM. Therefore, we inserted the Working Memory Index of WMS-III as independent variable and the MMN to the deviants that were statistically significant from the previous analysis as dependent variables.

In order to explore the different contributions of the two principal subtests of WMS-III on the observed relationship, we performed a further MANCOVA, inserting musical training group as independent grouping variable, the two principal 
subtests of the WMS-III (Spatial Span and Letter Number Sequencing) as independent continuous variables and the MMN amplitudes to each deviant as dependent variables. Again, we controlled for age by entering it into the model as covariate.

Results are reported with Greenhouse-Geisser corrected test values. Bonferroni adjustment was used for post-hoc tests. For all the significant findings, we reported effect sizes as indexed by partial eta-squared $(\eta 2 p)$.

\section{Acknowledgments}

We wish to thank the following persons for their help with MEG/EEG data collection: Benjamin Gold, David Ellison, Anja Thiede, Chao Liu, Suvi Lehto, Dr. Jyrki Mäkelä, Dr. Simo Monto. We were also assisted in collecting the psychological data by the following persons: Brigitte Bogert, Johanna Nohrström, Taru Numminen-Kontti, and Mikko Heimölä. Finally, we would like to acknowledge the financial support of the Academy of Finland (project number 133673), the 3-years grant of the University of Helsinki and the Danish National Research Foundation (project number DNRF117).

\section{References}

Acton, G. S., \& Schroeder, D. H. (2001). Sensory discrimination as related to general intelligence. Intelligence, 29(3), 263-271. https://doi.org/10.1016/S0160-2896(01)00066-6

Ahveninen, J., Jääskeläinen, I. P., Pekkonen, E., Hallberg, A., Hietanen, M., Mäkelä, R., ... Sillanaukee, P. (1999). Suppression of mismatch negativity by backward masking predicts impaired working-memory performance in alcoholics. Alcoholism, Clinical and Experimental Research, 23(9), 1507-14. https://doi.org/10.1111/j.15300277.1999.tb04674.x 
Allen, R. J., Hitch, G. J., \& Baddeley, A. D. (2009). Cross-modal binding and working memory. Visual Cogn, 17(1 \& 2), 83-102. https://doi.org/10.1080/13506280802281386

Arnell, K. M. (2006). Visual, auditory, and cross-modality dual-task costs: electrophysiological evidence for an amodal bottleneck on working memory consolidation. Perception \& Psychophysics, 68(3), 447-457. https://doi.org/10.3758/BF03193689

Atienza, M., Cantero, J. L., \& Dominguez-Marin, E. (2002). Mismatch negativity (MMN): An objective measure of sensory memory and long-lasting memories during sleep. International Journal of Psychophysiology, 46(3), 215225. https://doi.org/10.1016/S0167-8760(02)00113-7

Awh, E., Vogel, E. K., \& Oh, S. H. (2006). Interactions between attention and working memory. Neuroscience, 139(1), 201-208. https://doi.org/10.1016/j.neuroscience.2005.08.023

Baddeley, A. (1992). Working memory. Science, 255(5044), 556-559. https://doi.org/10.1126/science.1736359

Baddeley, A. (2000). The episodic buffer: A new component of working memory? Trends in Cognitive Sciences. https://doi.org/10.1016/S1364-6613(00)01538-2

Baddeley, A. (2012). Working memory: theories, models, and controversies. Annual Review of Psychology, 63, 1-29. https://doi.org/10.1146/annurev-psych-120710-100422

Baddeley, A., \& Hitch, G. (1974). Working memory. Psychology of Learning and Motivation, 8, 47-89. https://doi.org/10.1016/S0079-7421(08)60452-1

Bonetti, L., \& Costa, M. (2016). Intelligence and musical mode preference. Empirical Studies of the Arts, 34(2), 160176. https://doi.org/10.1177/0276237416628907

Bonetti, L., \& Costa, M. (2017a). Musical mode and visual-spatial cross-modal associations in infants and adults. Musicae Scientiae, 102986491770500. https://doi.org/10.1177/1029864917705001

Bonetti, L., \& Costa, M. (2017b). Pitch-verticality and pitch-size cross-modal interactions. Psychology of Music, 30573561771073. https://doi.org/10.1177/0305735617710734

Bonetti, L., Haumann, N. T., Vuust, P., Kliuchko, M., \& Brattico, E. (2017). Risk of depression enhances auditory Pitch discrimination in the brain as indexed by the mismatch negativity. Clinical Neurophysiology, 128(10), 1923-1936. https://doi.org/10.1016/j.clinph.2017.07.004

Brattico, E., Näätänen, R., \& Tervaniemi, M. (2001). Context effects on pitch perception in musicians and nonmusicians: Evidence from event-related potential recordings. Music Perception, 19(2), 199-222. https://doi.org/10.1525/mp.2001.19.2.199

Brattico, E., Pallesen, K. J., Varyagina, O., Bailey, C., Anourova, I., Järvenpää, M., ... Tervaniemi, M. (2009). Neural discrimination of nonprototypical chords in music experts and laymen: an MEG study. Journal of Cognitive Neuroscience, 21(11), 2230-2244. https://doi.org/10.1162/jocn.2008.21144

Brattico, E., Tervaniemi, M., Näätänen, R., \& Peretz, I. (2006). Musical scale properties are automatically processed in the human auditory cortex. Brain Research, 1117(1), 162-174. https://doi.org/10.1016/j.brainres.2006.08.023

Cashdollar, N., Ruhnau, P., Weisz, N., \& Hasson, U. (2016). The role of working memory in the probabilistic inference of future sensory events. Cerebral Cortex, 1-15. https://doi.org/10.1093/cercor/bhw138

Cheour, M., Leppänen, P. H., \& Kraus, N. (2000). Mismatch negativity (MMN) as a tool for investigating auditory discrimination and sensory memory in infants and children. Clinical Neurophysiology : Official Journal of the International Federation of Clinical Neurophysiology, 111(1), 4-16.

Cheour, M., Shestakova, A., Alku, P., Ceponiene, R., \& Naatanen, R. (2002). Mismatch negativity shows that 3-6 yearold children can learn discriminate non-native speech sounds within two months. Neurosci Lett, 325, $187-190$. https://doi.org/10.1016/S0304-3940(02)00269-0

Chun, M. M. (2011). Visual working memory as visual attention sustained internally over time. Neuropsychologia, 
49(6), 1407-1409. https://doi.org/10.1016/j.neuropsychologia.2011.01.029

Constantinidis, C. (2016). A Code for Cross-Modal Working Memory. Neuron. https://doi.org/10.1016/j.neuron.2015.12.033

Courtney, S. M. (1998). An Area Specialized for Spatial Working Memory in Human Frontal Cortex. Science, 279(5355), 1347-1351. https://doi.org/10.1126/science.279.5355.1347

Courtney, S. M., Petit, L., Maisog, J. M., Ungerleider, L. G., \& Haxby, J. V. (1998). An area specialized for spatial working memory in human frontal cortex. Science, 279(5355), 1347-1351.

https://doi.org/10.1126/science.279.5355.1347

Courtney, S. M., Petit, L., Maisog, J. M., Ungerleider, L. G., Haxby, J. V, Funahashi, S., ... Gottlieb, J. P. (1998). An area specialized for spatial working memory in human frontal cortex. Science (New York, N.Y.), 279(5355), $1347-51$. https://doi.org/10.1126/science.279.5355.1347

Cowan, N. (1999). An embedded-processes model of working memory. Models of Working Memory: Mechanisms of Active Maintenance and Executive Control. https://doi.org/10.1017/S0140525X01003922

Cowan, N. (2008). 2.03 Sensory Memory. Memory, (1960), 23-32. https://doi.org/10.1016/B978-012370509-9.001728

Cowan, N. (2010). Sensory and Immediate Memory. In Encyclopedia of Consciousness (pp. 327-339). https://doi.org/10.1016/B978-012373873-8.00048-7

Crowe, S. F. (2000). Does the Letter Number Sequecing Task Measure Anything More Than Digit Span? Assessment, 7(2), 113-117. https://doi.org/0803973233

Curtis, C. E. (2006). Prefrontal and parietal contributions to spatial working memory. Neuroscience, 139(1), 173-180. https://doi.org/10.1016/j.neuroscience.2005.04.070

Deary, I. J. (2012). Intelligence. Annual Review of Psychology, 63, 453-82. https://doi.org/10.1146/annurev-psych120710-100353

Deary, I. J., Bell, P. J., Bell, A. J., Campbell, M. L., \& Fazal, N. D. (2004). Sensory discrimination and intelligence: Testing Spearman's other hypothesis. American Journal of Psychology, 117(1), 1-18. https://doi.org/10.2307/1423593

Deouell, L. Y. (2007). The frontal generator of the mismatch negativity revisited. Journal of Psychophysiology, 21(3-4), 188-203. https://doi.org/10.1027/0269-8803.21.34.188

Duarte, A., Hearons, P., Jiang, Y., Delvin, M. C., Newsome, R. N., \& Verhaeghen, P. (2013). Retrospective attention enhances visual working memory in the young but not the old: An ERP study. Psychophysiology, 50(5), 465-476. https://doi.org/10.1111/psyp.12034

Engle, R. W. (2002). Working memory capacity as executive attention. Current Directions in Psychological Science, 11(1), 19-23. https://doi.org/10.1111/1467-8721.00160

Engle, R. W., \& Kane, M. J. (2004). Executive Attention, Working Memory Capacity, and a Two-Factor Theory of Cognitive Control. The Psychology of Learning and Motivation: Advances in Research and Theory, 145-199. https://doi.org/10.1016/S0079-7421(03)44005-X

Escera, C., Alho, K., Winkler, I., \& Näätänen, R. (1998). Neural Mechanisms of Involuntary Attention to Acoustic Novelty and Change. Journal of Cognitive Neuroscience, 10(5), 590-604. https://doi.org/10.1162/089892998562997

Fahy, F. J. (1997). International standards for the determination of sound power levels of sources using sound intensity measurement: an exposition. Applied Acoustics, 50(2), 97-109. https://doi.org/10.1016/S0003$682 \times(96) 00043-6$

Förstl, H., Tulving, E., \& Craik, F. I. M. (2006). The Oxford handbook of memory. Zeitschrift für Psychiatrie, Psychologie und Psychotherapie (Vol. 54). https://doi.org/10.1024/1661-4747.54.1.68a 
Fougnie, D. (2008). The relationship between attention and working memory. New Research on Short-Term Memory. https://doi.org/10.3389/conf.fnhum.2011.207.00576

Garza Villarreal, E. A., Brattico, E., Leino, S., Østergaard, L., \& Vuust, P. (2011). Distinct neural responses to chord violations: A multiple source analysis study. Brain Research, 1389, 103-114. https://doi.org/10.1016/j.brainres.2011.02.089

Gifford, R., Steg, L., \& Reser, J. (2011). Environmental psychology. IAAP Handbooks of Applied Psychology, 440-470. https://doi.org/10.1007/SpringerReference_29557

Gross, J., Baillet, S., Barnes, G. R., Henson, R. N., Hillebrand, A., Jensen, O., ... Schoffelen, J. M. (2013). Good practice for conducting and reporting MEG research. Neurolmage. https://doi.org/10.1016/j.neuroimage.2012.10.001

Haenschel, C., Vernon, D. J., Dwivedi, P., Gruzelier, J. H., \& Baldeweg, T. (2005). Event-Related Brain Potential Correlates of Human Auditory Sensory Memory-Trace Formation. The Journal of Neuroscience, 25(45), 1049410501. https://doi.org/10.1523/jneurosci.1227-05.2005

Hahn, B., Hollingworth, A., Robinson, B. M., Kaiser, S. T., Leonard, C. J., Beck, V. M., ... Gold, J. M. (2012). Control of working memory content in schizophrenia. Schizophrenia Research, 134(1), 70-75. https://doi.org/10.1016/j.schres.2011.10.008

Hansen, M., Wallentin, M., \& Vuust, P. (2013). Working memory and musical competence of musicians and nonmusicians. Psychology of Music, 41(6), 779-793. https://doi.org/10.1177/0305735612452186

Haumann, N. T., Parkkonen, L., Kliuchko, M., Vuust, P., \& Brattico, E. (2016). Comparing the Performance of Popular MEG/EEG Artifact Correction Methods in an Evoked-Response Study. Computational Intelligence and Neuroscience, 1-10. https://doi.org/10.1155/2016/7489108

Hoelzle, J. B., Nelson, N. W., \& Smith, C. a. (2011). Comparison of Wechsler Memory Scale-Fourth Edition (WMS-IV) and Third Edition (WMS-III) dimensional structures: improved ability to evaluate auditory and visual constructs. Journal of Clinical and Experimental Neuropsychology, 33(3), 283-291. https://doi.org/10.1080/13803395.2010.511603

Javitt, D. C., Doneshka, P., Grochowski, S., \& Ritter, W. (1995). Impaired mismatch negativity generation reflects widespread dysfunction of working memory in schizophrenia. Archives of General Psychiatry, 52(7), 550-558. https://doi.org/10.1001/archpsyc.1995.03950190032005

Kaufman, A. S., \& Lichtenberger, E. O. (2006). Assessing adolescent and adult intelligence (3rd ed.). Assessing adolescent and adult intelligence (3rd ed.).

Kaufman, J. C., Plucker, J. a, \& Baer, J. (2008). Essentials of creativity assessment. Essentials of psychological assessment series.

Kimura, M. (2012). Visual mismatch negativity and unintentional temporal-context-based prediction in vision. International Journal of Psychophysiology. https://doi.org/10.1016/j.ijpsycho.2011.11.010

Kliuchko, M., Heinonen-Guzejev, M., Vuust, P., Tervaniemi, M., \& Brattico, E. (2016). A window into the brain mechanisms associated with noise sensitivity. Sci Rep, 6, 39236. doi:10.1038/srep39236

Koelsch, S., Schro, C. A. E., Tervaniemi, M., \& Schröger, E. (1999). Superior pre-attentive auditory processing in musicians. Neuroreport, 10(6), 1309-13. https://doi.org/10.1097/00001756-199904260-00029

Kraus, N., McGee, T., Carrell, T. D., \& Sharma, A. (1995). Neurophysiologic bases of speech discrimination. Ear and Hearing, 16(1), 19-37. https://doi.org/10.1097/00003446-199502000-00003

Kumar, T. S. Anand, \& Newport, J. K. (2015). Routledge International Handbook of Memory Studies. Disaster Prevention and Management, 14(2), 570. https://doi.org/10.1108/09653560510595173

Lichtenberger, E. O., \& Smith, D. R. (2005). Essentials of WIAT-II and KTEA-II assessment. Essentials of psychological assessment series.

Lijffijt, M., Lane, S. D., Meier, S. L., Boutros, N. N., Burroughs, S., Steinberg, J. L., ... Swann, A. C. (2009). P50, N100, and 
P200 sensory gating: Relationships with behavioral inhibition, attention, and working memory.

Psychophysiology, 46(5), 1059-1068. https://doi.org/10.1111/j.1469-8986.2009.00845.x

Lindenberger, U., \& Baltes, P. B. (1994). Sensory functioning and intelligence in old age: a strong connection. Psychology and Aging, 9(3), 339-55. https://doi.org/10.1037/0882-7974.9.3.339

Makeig, S., J. Bell., A., Jung, T.-P., \& Sejnowski, T. J. (1996). Independent Component Analysis of Electroencephalographic Data. Advances in Neural Information Processing Systems, 8(3), 145-151. https://doi.org/10.1109/ICOSP.2002.1180091

Mccabe, D. P., Henry L. Roediger III, Mcdaniel, M. a, Balota, D. A., \& Hambrick, D. Z. (2010). The Relationship Between Working Memory Capacity and Executive Functioning: Evidence for a Common Executive Attention Construct. Neuropsychology, 24(2), 222-243. https://doi.org/10.1037/a0017619

McKone, E., \& Dennis, C. (2000). Short-term implicit memory: visual, auditory, and cross-modality priming. Psychonomic Bulletin \& Review, 7(2), 341-346. https://doi.org/10.3758/BF03212991

McNab, F., \& Klingberg, T. (2008). Prefrontal cortex and basal ganglia control access to working memory. Nature Neuroscience, 11(1), 103-107. https://doi.org/10.1038/nn2024

Moreno, S., Marques, C., Santos, A., Santos, M., Castro, S. L., \& Besson, M. (2009). Musical training influences linguistic abilities in 8-year-old children: More evidence for brain plasticity. Cerebral Cortex, 19(3), 712-723. https://doi.org/10.1093/cercor/bhn120

Näätänen, R. (1992). Attention and brain function. London: Psychology Press.

Näätänen, R., Astikainen, P., Ruusuvirta, T., \& Huotilainen, M. (2010). Automatic auditory intelligence: An expression of the sensory-cognitive core of cognitive processes. Brain Research Reviews. https://doi.org/10.1016/j.brainresrev.2010.03.001

Näätänen, R., Gaillard, A. W. K., \& Mäntysalo, S. (1978). Early selective-attention effect on evoked potential reinterpreted. Acta Psychologica, 42(4), 313-329. https://doi.org/10.1016/0001-6918(78)90006-9

Näätänen, R., Kujala, T., Kreegipuu, K., Carlson, S., Escera, C., Baldeweg, T., \& Ponton, C. (2011). The mismatch negativity: An index of cognitive decline in neuropsychiatric and neurological diseases and in ageing. In Brain (Vol. 134, pp. 3432-3450). https://doi.org/10.1093/brain/awr064

Näätänen, R., Paavilainen, H., Titinen, H., Jiang, D., \& Alho, K. (1993). Attention and mismatch negativity. Psychophysiology, 30(5), 436-450. https://doi.org/10.1111/j.1469-8986.1993.tb02067.x

Näätänen, R., Paavilainen, P., Tiitinen, H., Jiang, D., \& Alho, K. (1993). Attention and mismatch negativity. Psychophysiology, 30(5), 436-450. https://doi.org/10.1111/j.1469-8986.1993.tb02067.x

Näätänen, R., Sussman, E. S., Salisbury, D., \& Shafer, V. L. (2014). Mismatch negativity (MMN) as an index of cognitive dysfunction. Brain Topography. https://doi.org/10.1007/s10548-014-0374-6

Oberauer, K., \& Hein, L. (2012). Attention to Information in Working Memory. Current Directions in Psychological Science, 21(3), 164-169. https://doi.org/10.1177/0963721412444727

Onton, J., Delorme, A., \& Makeig, S. (2005). Frontal midline EEG dynamics during working memory. Neurolmage, 27(2), 341-356. https://doi.org/10.1016/j.neuroimage.2005.04.014

Oostenveld, R., Fries, P., Maris, E., \& Schoffelen, J. (2011). FieldTrip: Open source software for advanced analysis of MEG, EEG, and invasive electrophysiological data. Computational Intelligence and Neuroscience : CIN, $2011,1-9$. https://doi.org/:10.1155/2011/156869

Opitz, B., Rinne, T., Mecklinger, A., von Cramon, D. Y., \& Schröger, E. (2002). Differential Contribution of Frontal and Temporal Cortices to Auditory Change Detection: fMRI and ERP Results. Neurolmage, 15(1), 167-174. https://doi.org/10.1006/nimg.2001.0970

Park, S., \& McTigue, K. (1997). Working memory and the syndromes of schizotypal personality. Schizophrenia 
Research, 26(2-3), 213-220. https://doi.org/10.1016/S0920-9964(97)00051-0

Pasternak, T., \& Greenlee, M. W. (2005). Working memory in primate sensory systems. Nature Reviews. Neuroscience, 6(2), 97-107. https://doi.org/10.1038/nrn1603

Peltola, M. S., Kujala, T., Tuomainen, J., Ek, M., Aaltonen, O., \& Näätänen, R. (2003). Native and foreign vowel discrimination as indexed by the mismatch negativity (MMN) response. Neuroscience Letters, 352(1), 25-28. https://doi.org/10.1016/j.neulet.2003.08.013

Potkin, S. G., Turner, J. A., Brown, G. G., McCarthy, G., Greve, D. N., Glover, G. H., ... Lim, K. O. (2009). Working memory and DLPFC inefficiency in schizophrenia: The FBIRN study. Schizophrenia Bulletin, 35(1), 19-31. https://doi.org/10.1093/schbul/sbn162

Restuccia, D., Della Marca, G., Marra, C., Rubino, M., \& Valeriani, M. (2005). Attentional load of the primary task influences the frontal but not the temporal generators of mismatch negativity. Cognitive Brain Research, 25(3), 891-899. https://doi.org/10.1016/j.cogbrainres.2005.09.023

Rinne, T., Alho, K., Ilmoniemi, R. J., Virtanen, J., \& Näätänen, R. (2000). Separate Time Behaviors of the Temporal and Frontal Mismatch Negativity Sources. Neurolmage, 12(1), 14-19. https://doi.org/10.1006/nimg.2000.0591

Ruzzoli, M., Pirulli, C., Brignani, D., Maioli, C., \& Miniussi, C. (2012). Sensory memory during physiological aging indexed by mismatch negativity (MMN). Neurobiology of Aging, 33(3). https://doi.org/10.1016/j.neurobiolaging.2011.03.021

Sligte, I. G., Vandenbroucke, A. R. E., Scholte, H. S., \& Lamme, V. A. F. (2010). Detailed sensory memory, sloppy working memory. Frontiers in Psychology, (OCT). https://doi.org/10.3389/fpsyg.2010.00175

Suchan, B., Linnewerth, B., Köster, O., Daum, I., \& Schmid, G. (2006). Cross-modal processing in auditory and visual working memory. Neurolmage, 29(3), 853-858. https://doi.org/10.1016/j.neuroimage.2005.08.014

Takegata, R., Brattico, E., Tervaniemi, M., Varyagina, O., Näätänen, R., \& Winkler, I. (2005). Preattentive representation of feature conjunctions for concurrent spatially distributed auditory objects. Cognitive Brain Research, 25(1), 169-179. https://doi.org/10.1016/j.cogbrainres.2005.05.006

Talamini, F., Carretti, B., \& Grassi, M. (2016). The Working Memory of Musicians and Nonmusicians. Music Perception, 34(2), 183-191. https://doi.org/10.1525/mp.2016.34.2.183

Taulu, S., \& Hari, R. (2009). Removal of magnetoencephalographic artifacts with temporal signal-space separation: demonstration with single-trial auditory-evoked responses. Human Brain Mapping, 30(5), 1524-1534. https://doi.org/10.1002/hbm.20627

Tervaniemi, M. (2001). Superior Formation of Cortical Memory Traces for Melodic Patterns in Musicians. Learning \& Memory, 8(5), 295-300. https://doi.org/10.1101/Im.39501

Tervaniemi, M. (2006). Musical Sound Processing in the Human Brain. Annals of the New York Academy of Sciences, 930(1), 259-272. https://doi.org/10.1111/j.1749-6632.2001.tb05737.x

Tervaniemi, M., Just, V., Koelsch, S., Widmann, A., \& Schröger, E. (2005). Pitch discrimination accuracy in musicians vs nonmusicians: An event-related potential and behavioral study. Experimental Brain Research, 161(1), 1-10. https://doi.org/10.1007/s00221-004-2044-5

Tremblay, K., Kraus, N., Carrell, T. D., \& McGee, T. (1997). Central auditory system plasticity: Generalization to novel stimuli following listening training. Journal of the Acoustical Society of America, 102(6), 3762-3773. https://doi.org/10.1121/1.420139

Tse, C. Y., \& Penney, T. B. (2008). On the functional role of temporal and frontal cortex activation in passive detection of auditory deviance. Neurolmage, 41(4), 1462-1470. https://doi.org/10.1016/j.neuroimage.2008.03.043

Vuust, P., Brattico, E., Glerean, E., Seppänen, M., Pakarinen, S., Tervaniemi, M., \& Näätänen, R. (2011). New fast 
mismatch negativity paradigm for determining the neural prerequisites for musical ability. Cortex, 47(9), 10911098. https://doi.org/10.1016/j.cortex.2011.04.026

Vuust, P., Brattico, E., Seppänen, M., Näätänen, R., \& Tervaniemi, M. (2012). The sound of music: Differentiating musicians using a fast, musical multi-feature mismatch negativity paradigm. Neuropsychologia, 50(7), 14321443. https://doi.org/10.1016/j.neuropsychologia.2012.02.028

Vuust, P., Liikala, L., Näätänen, R., Brattico, P., \& Brattico, E. (2016). Comprehensive auditory discrimination profiles recorded with a fast parametric musical multi-feature mismatch negativity paradigm. Clinical Neurophysiology, 127(4), 2065-2077. https://doi.org/10.1016/j.clinph.2015.11.009

Wacongne, C., Changeux, J.-P., \& Dehaene, S. (2012). A Neuronal Model of Predictive Coding Accounting for the Mismatch Negativity. Journal of Neuroscience, 32(11), 3665-3678. https://doi.org/10.1523/JNEUROSCI.500311.2012

Wechsler, D. (1997). Wechsler Memory Scale- (Third Ed.). The Psychological Corporation.

Wilde, N. J., \& Strauss, E. (2002). Functional equivalence of WAIS- III/WMS-III digit and spatial span under forward and backward recall conditions. The Clinical Neuropsychologist, 16(3), 322-330. https://doi.org/10.1076/clin.16.3.322.13858

Winkler, I., \& Czigler, I. (2012). Evidence from auditory and visual event-related potential (ERP) studies of deviance detection (MMN and vMMN) linking predictive coding theories and perceptual object representations. International Journal of Psychophysiology. https://doi.org/10.1016/j.ijpsycho.2011.10.001

Winkler, I., Kujala, T., Tiitinen, H., Sivonen, P., Alku, P., Lehtokoski, a, ... Näätänen, R. (1999). Brain responses reveal the learning of foreign language phonemes. Psychophysiology, 36(5), 638-642. https://doi.org/10.1111/14698986.3650638 


\section{Figure legends}

Figure 1 Grand averaged MMN recorded at the frontal ROI sensors for each participant and for each deviant type represented in the musical multi-feature paradigm. The waveforms have been obtained subtracting the evoked responses to the standard stimuli to the evoked responses to the deviant stimuli.

Figure $2 \mathrm{MMN}$ to intensity and working memory: waveforms figure and Pearson's correlations between MMN and WM showed along all the MEG sensors.

Figure 3 MMN to slide and working memory: waveforms figure and Pearson's correlations between MMN and WM showed along all the MEG sensors.

Figure 4 Regressions for musicians and non-musicians between the independent variable Working Memory Index and the dependent variable frontal MMN to intensity deviant.

Figure 5 Regressions for musicians and non-musicians between the independent variable Working Memory Index and the dependent variable frontal MMN to slide deviant.

Figure 6 Alberti bass stimulus used in the musical multi-feature paradigm. Each tone (except for the rhythm deviant) had a duration of $200 \mathrm{~ms}$. The tones were presented with an inter-stimulus interval (ISI) of 5 ms.

Figure 7 Clusters of sensors used in the analyses. 


\section{Figures}

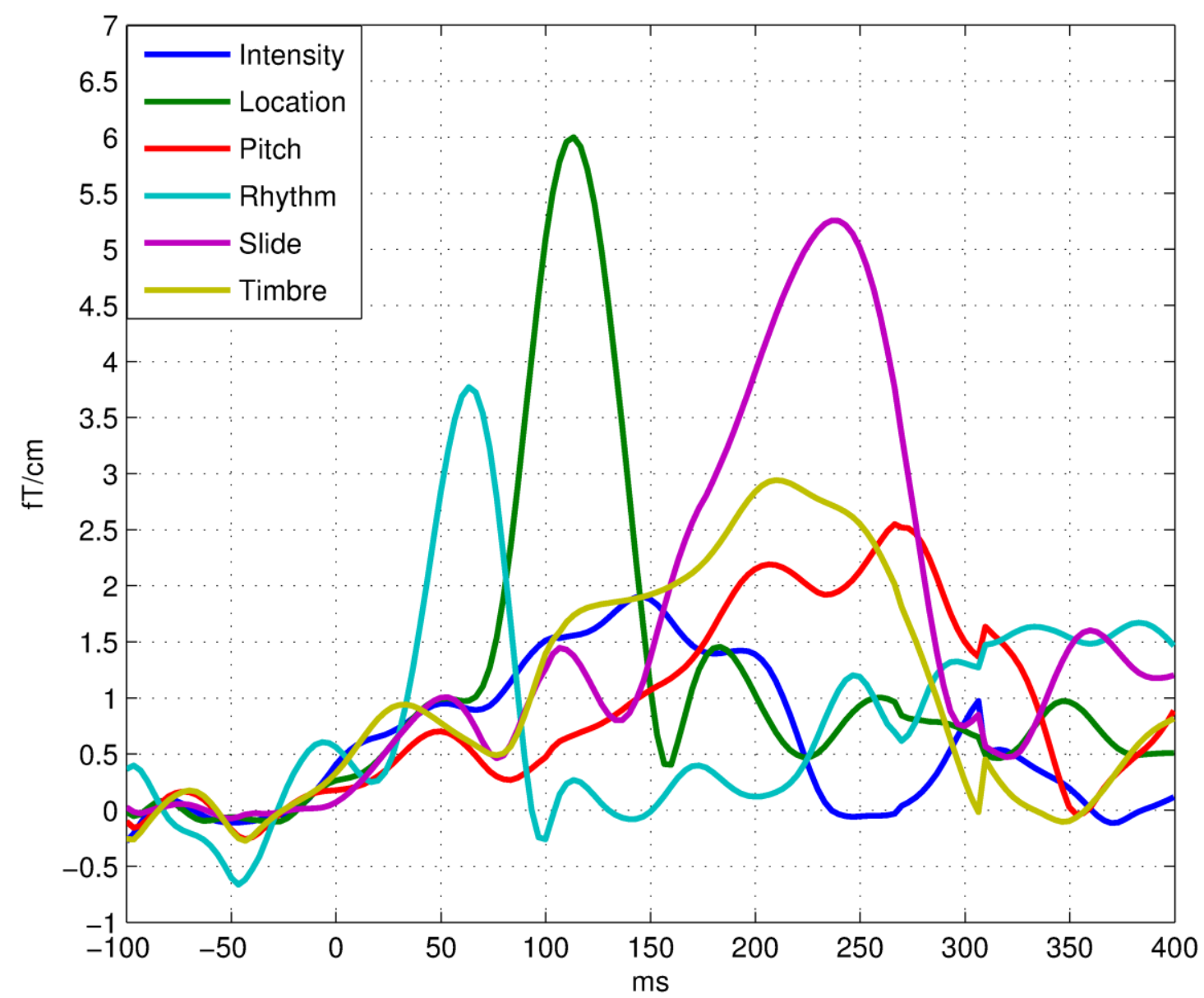

Figure 1 Grand averaged MMN recorded at the frontal ROI sensors for each participant and for each deviant type represented in the musical multi-feature paradigm. The waveforms have been obtained subtracting the evoked responses to the standard stimuli to the evoked responses to the deviant stimuli. 


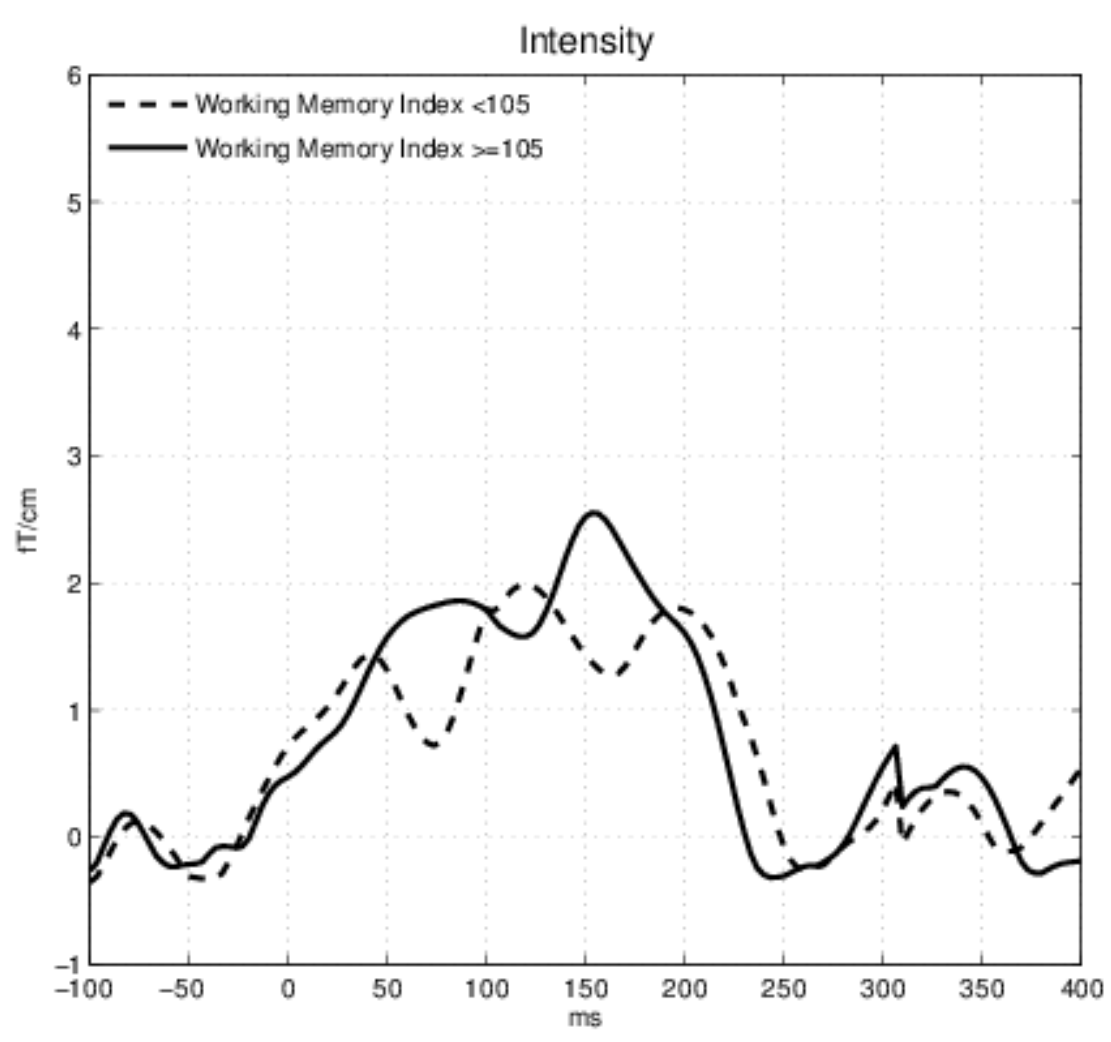

a) Frontal ROI grand average MMN to the intensity deviant for all participants, showed in relation to two different Working Memory Index score groups. One-hundred and five was the 50th percentile of the scores reported by the participants in our study (the dichotomy resulting into these two waves has been used for illustration purposes and have not been employed in the statistical analysis).

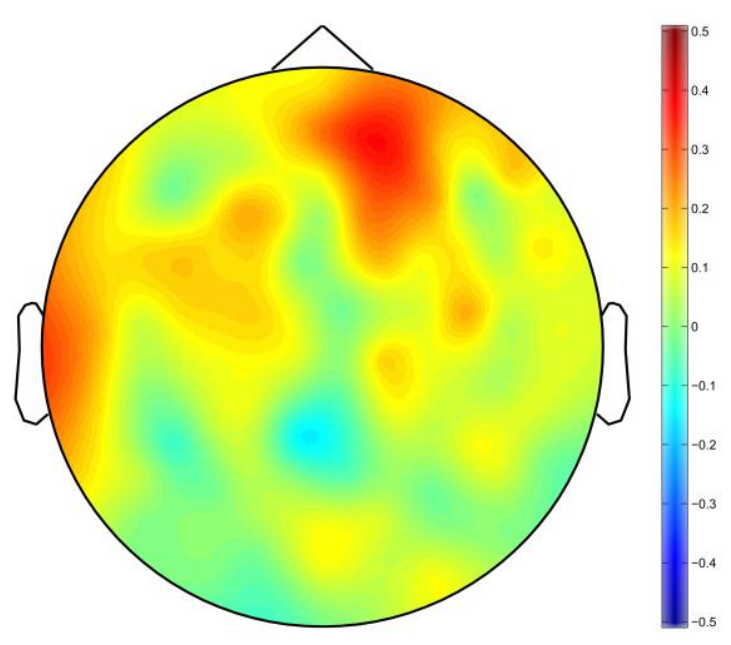

b) Pearson's correlation maps between MMN responses to intensity deviant averaged in an interval of \pm 15 ms around the maximal peak amplitudes and Working Memory Index scores along all MEG sensors. 


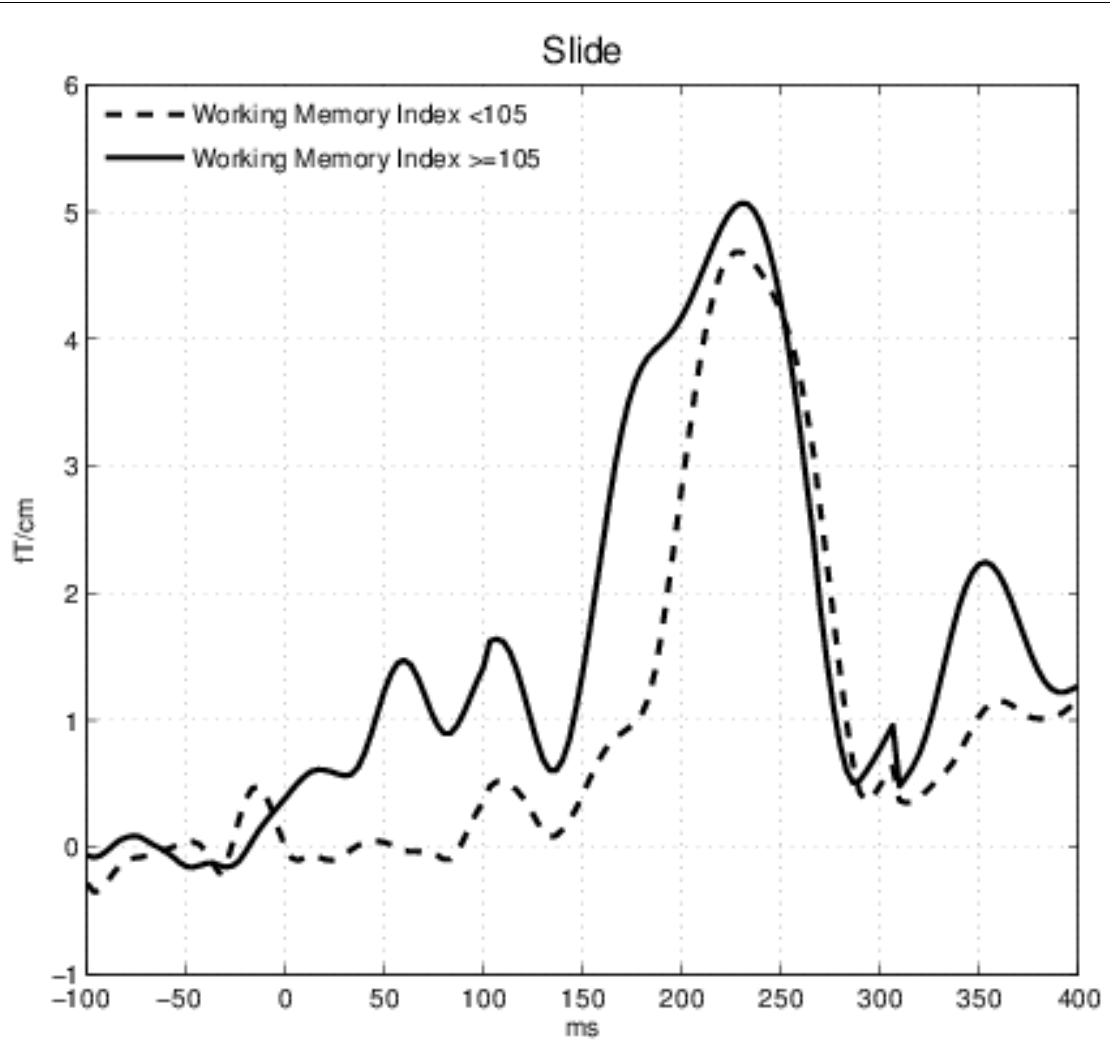

a) Frontal ROI grand average MMN to the slide deviant for all participants, showed in relation to two different Working Memory Index score groups. One-hundred and five was the 50th percentile of the scores reported by the participants in our study (the dichotomy resulting into these two waves has been used for illustration purposes and have not been employed in the statistical analysis). 


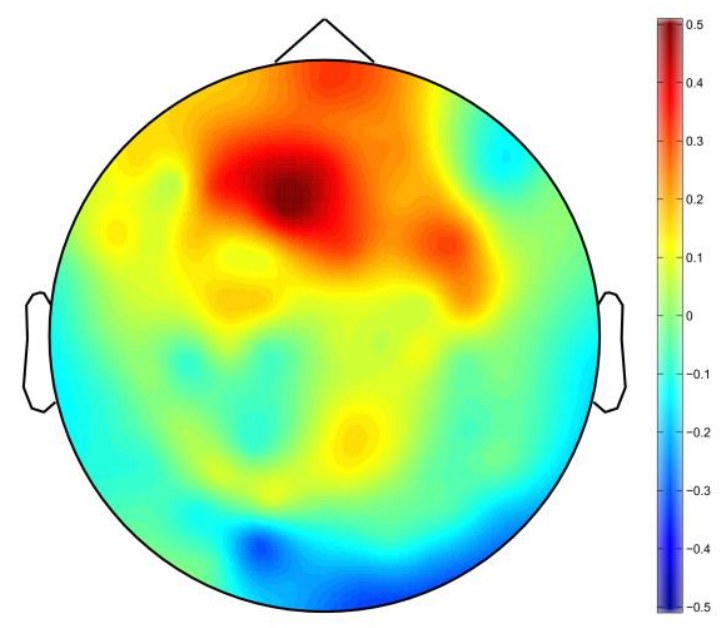

b) Pearson's correlation maps between $M M N$ responses to slide deviant averaged in an interval of $\pm 15 \mathrm{~ms}$ around the maximal peak amplitudes and Working Memory Index scores along all MEG sensors, considering musician sample only.

Figure $3 \mathrm{MMN}$ to slide and working memory: waveforms figure and Pearson's correlations between MMN and WM showed along the all MEG sensors.

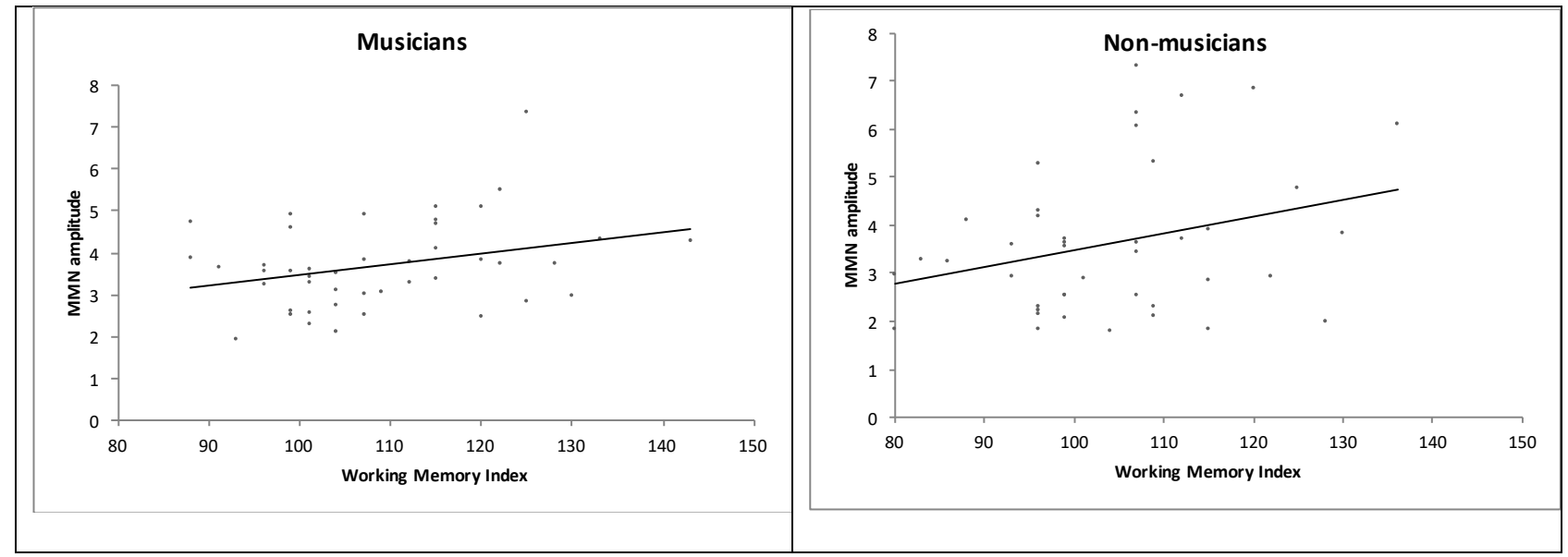

Figure 4 Regressions for musicians and non-musicians between the independent variable Working Memory Index and the dependent variable frontal MMN to intensity deviant. 


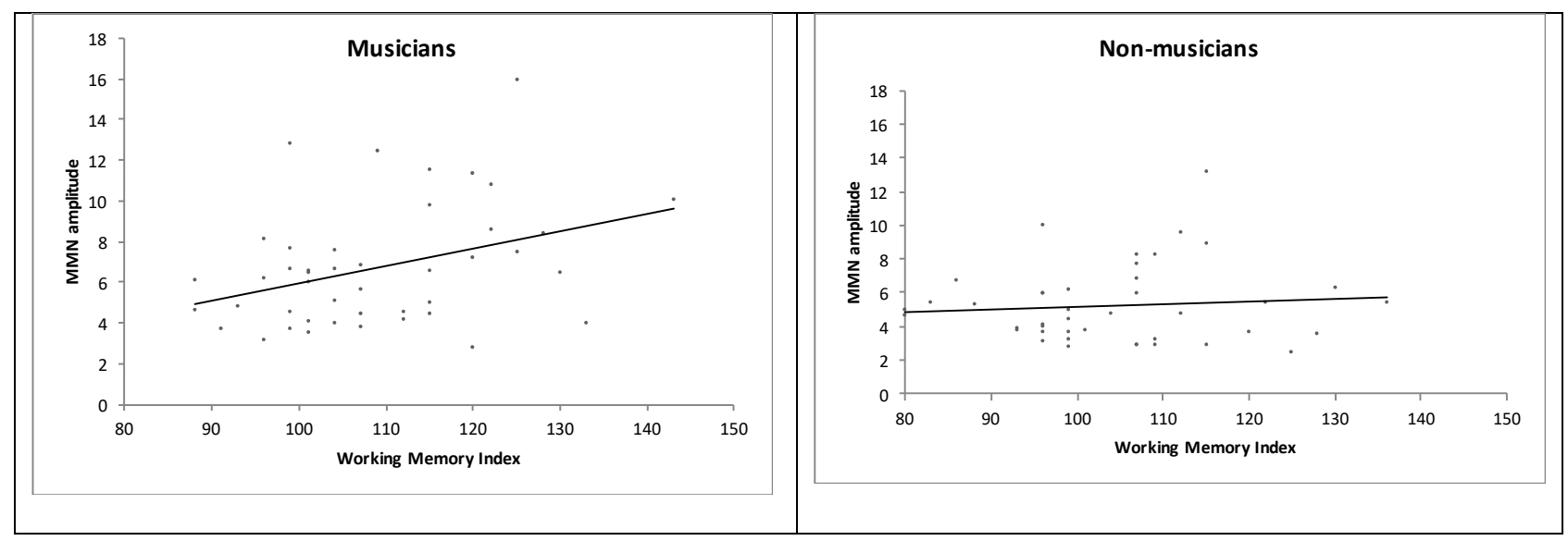

Figure 5 Regressions for musicians and non-musicians between the independent variable Working Memory Index and the dependent variable frontal MMN to slide deviant.

\section{Stimulus (Alberti Bass)}

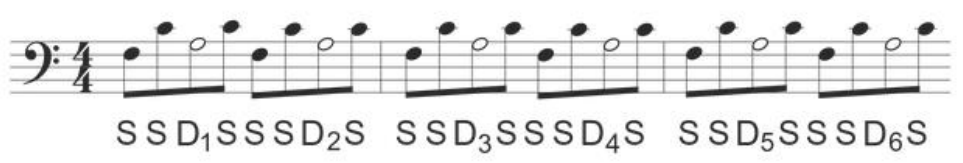

F-major Ab-minor D-minor....

S: Standard

D1: Pitch-deviant: 24 cents lower

$D_{2}$ : Timbre-deviant: filtered, having an 'old time radio' effect

D3: Location-deviant: slightly shifted to the left

D4: Intensity-deviant: $6 \mathrm{~dB}$ reduction

D5: Slide-deviant: sliding up from a whole note below

D6: Rhythm-deviant: 40 ms earlier

Figure 6 Alberti bass stimulus used in the musical multi-feature paradigm. Each tone (except for the rhythm deviant) had a duration of 200 ms. The tones were presented with an inter-stimulus interval (ISI) of 5 ms. 


\begin{tabular}{|c|c|}
\hline 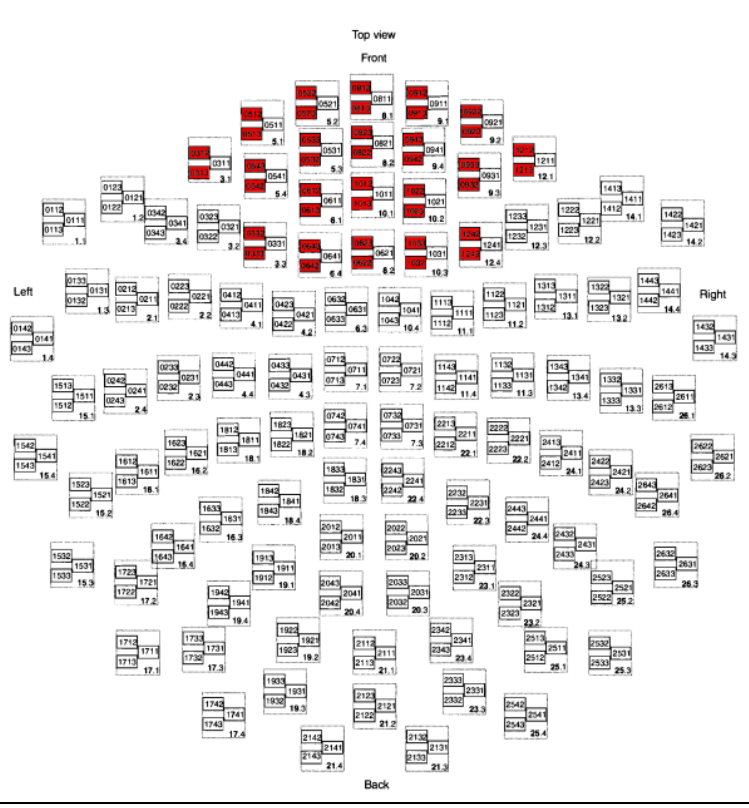 & 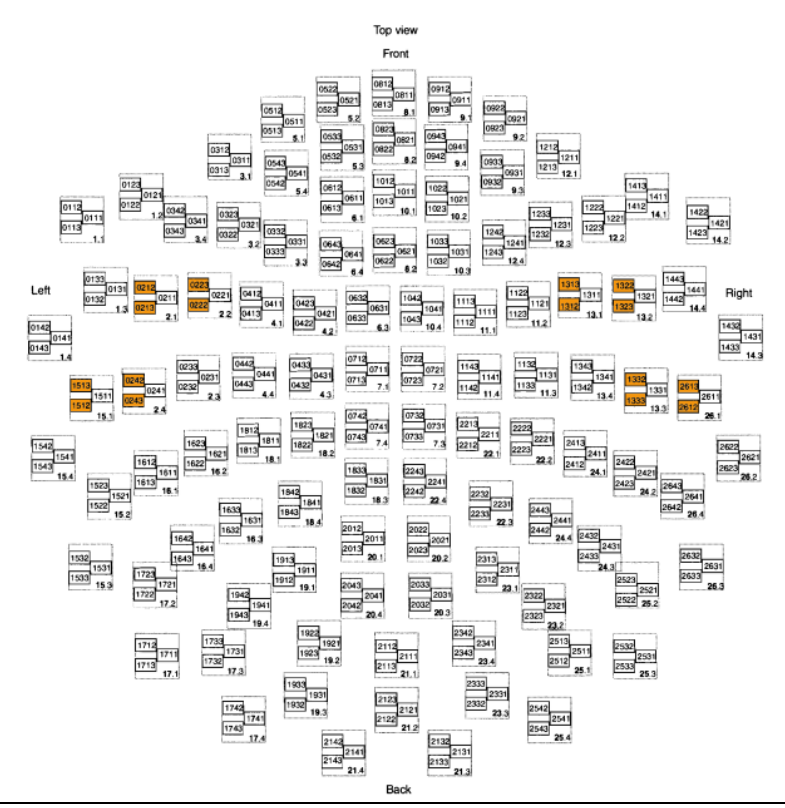 \\
\hline 7a) Sensors selected outlining one frontal ROI. & $\begin{array}{l}\text { 7b) Sensors selected outlining a temporal ROI involving } \\
\text { sensors within both left and right hemispheres. }\end{array}$ \\
\hline
\end{tabular}

Figure 7 Clusters of sensors used in the analyses.

\section{Tables}

\begin{tabular}{|c|c|c|c|c|}
\hline \multicolumn{5}{|c|}{ T-tests comparing evoked responses to standards and deviants } \\
\hline Deviant types & ROI & $\begin{array}{c}\text { Mean paired } \\
\text { differences (fT/cm) }\end{array}$ & $\boldsymbol{t}$ (85) & $p$ \\
\hline Intensity & Frontal & 2.37 & 20.63 & $<.001$ \\
\hline Localization & Frontal & 5.52 & 14.39 & $<.001$ \\
\hline Pitch & Frontal & 3.30 & 14.33 & $<.001$ \\
\hline Rhythm & Frontal & 4.51 & 22.17 & $<.001$ \\
\hline Slide & Frontal & 4.71 & 17.75 & $<.001$ \\
\hline Timbre & Frontal & 2.85 & 14.92 & $<.001$ \\
\hline Intensity & Temporal & 5.94 & 12.20 & $<$ \\
\hline
\end{tabular}




\begin{tabular}{|c|c|c|c|c|}
\hline Localization & Temporal & 20.67 & 11.05 & $<.001$ \\
\hline Pitch & Temporal & 11.33 & 10.33 & $<.001$ \\
\hline Rhythm & Temporal & 19.51 & 19.33 & $<.001$ \\
\hline Slide & Temporal & 17.52 & 17.11 & $<.001$ \\
\hline Timbre & Temporal & 9.78 & 10.98 & $<.001$ \\
\hline
\end{tabular}

Table 1 Independent t-tests comparing grand average MMN and standard responses for each participant and each deviant type at both frontal and temporal ROIs.

\begin{tabular}{|c|c|c|c|}
\hline \multicolumn{2}{|c|}{ MANCOVA between-subject effects of WM on MMN to the $\mathbf{6}$ type deviants of mumufe paradigm } \\
\hline Deviant & $\mathrm{F}(1,85)$ & $p$ & .09 \\
\hline Intensity & 7.95 & .006 & .008 \\
\hline Localization & .69 & .41 & .002 \\
\hline Pitch & .14 & .43 & .008 \\
\hline Rhythm & .64 & .02 & .06 \\
\hline Slide & 4.93 & .62 & .003 \\
\hline
\end{tabular}

Table 2 Tests of between-subject effects obtained from the MANCOVA involving the frontal ROI averaged MMN to each deviant as dependent variables and the WM score as independent variable. In bold the significant features.

Regressions showing the effect of the WM on the MMN to intensity deviant

\begin{tabular}{|c|c|c|c|}
\hline Musical training group & $F$ & $p$ & \\
\hline Musicians & $\mathrm{F}(1,43)=4.19$ & .04 & .31 \\
\hline Non-musicians & $\mathrm{F}(1,41)=3.93$ & .05 & .30 \\
\hline
\end{tabular}


Table 3 Regressions involving separately musicians and non-musicians between the independent variable Working Memory Index and the dependent variable $M M N$ to intensity deviant.

\begin{tabular}{|c|c|c|c|}
\hline \multicolumn{2}{|c|}{ Regressions showing the effect of the WM on the MMN to slide deviant } \\
\hline Musical training group & F & $p$ & .01 \\
\hline Musicians & $\mathbf{F}(\mathbf{1 , 4 3})=6.20$ & .60 & .36 \\
\hline Non-musicians & $\mathbf{F}(\mathbf{1 , 4 1 )}=.28$ & .08 \\
\hline
\end{tabular}

Table 4 Regressions involving separately musicians and non-musicians between the independent variable Working Memory Index and the dependent variable MMN to slide deviant.

\begin{tabular}{|c|c|c|c|}
\hline \multicolumn{2}{|c|}{ Spatial Span } \\
\hline Deviant & $F(1,85)$ & .02 & $\eta^{2}$ \\
\hline Intensity & 5.54 & .61 & .063 \\
\hline Localization & .26 & .90 & .001 \\
\hline Pitch & .02 & 53 & .005 \\
\hline Rhythm & .39 & .03 & .001 \\
\hline
\end{tabular}

Table 5. Tests of between-subject effects obtained from the MANCOVA performed for the averaged frontal ROI sensors, inserting the Spatial Span subtest as independent variable and the MMN to all deviants as dependent variables. 


\begin{tabular}{|c|c|c|c|c|c|}
\hline \multirow{3}{*}{ Group } & Sex & $\begin{array}{c}\text { Number of } \\
\text { participants }\end{array}$ & $\begin{array}{c}\text { Mean age of } \\
\text { participants (years) }\end{array}$ & $\begin{array}{c}\text { Mean length of } \\
\text { musical training } \\
\text { (years) }\end{array}$ & IQ \\
\hline \multirow{3}{*}{ Musicians } & Males & 20 & $30.7 \pm 9.8(19-45)$ & $10.1 \pm 10.5$ & $121.5 \pm 8.6$ \\
\cline { 2 - 6 } & Females & 24 & $26.6 \pm 7.4(20-44)$ & $7.8 \pm 6.1$ & $117.5 \pm 7.1$ \\
\cline { 2 - 6 } & Total & 44 & $28.5 \pm 8.7(19-45)$ & $9.2 \pm 8.1$ & $119.3 \pm 8.0$ \\
\hline \multirow{3}{*}{ Non- } & Males & 16 & $28.1 \pm 6.7(19-42)$ & $2.2 \pm 3.2$ & $118.2 \pm 8.0$ \\
\cline { 2 - 6 } & Females & 26 & $28.4 \pm 9.8(20-55)$ & $0.9 \pm 1.8$ & $116.5 \pm 7.5$ \\
\cline { 2 - 6 } & Total & 42 & $28.3 \pm 8.6(19-55)$ & $1.4 \pm 2.5$ & $117.2 \pm 7.7$ \\
\hline
\end{tabular}

Table 6. Means of participants' age, length of musical training and IQ according to musicianship and sex (the number after ' \pm ' refers to standard deviations, while the numbers in parenthesis express the age range of the participants).

\begin{tabular}{|l|c|c|}
\hline Scales & Musicians & Non-musicians \\
\hline Spatial Span & $11.77 \pm 3.20$ & $10.62 \pm 2.94$ \\
\hline Letter Number Sequencing & $12.05 \pm 2.69$ & $11.40 \pm 2.77$ \\
\hline Working Memory Index & $108.82 \pm 12.62$ & $104.12 \pm 13.01$ \\
\hline
\end{tabular}

Table 7 Working Memory Index and relative subscales measured through WMS-III for musicians and non-musicians. 